\title{
NLS EQUATION WITH COMPLEX POTENTIAL: MODES AND PSEUDO-MODES
}

\author{
@ G.L. Alfimov, A.O. Slobodyanyuk, D.A. Zezyulin \\ galfimov@yahoo.com
}

УДК 517.957.6

DOI: 10.33184/mnkuomsh2t-2021-10-06.5.

Недавно Ю. Коминис и соавторы [Хаос, солитоны и фракталы, bf 118, 222-233 (2019)] предположили, что нелинейное уравнение Шрёдингера (NLS) с комплексным потенциалом вида $W(x)=W_{1}(x)+$ $i C W_{1, x}(x)$, поддерживает непрерывные семейства локализованных нелинейных мод. Здесь $C \in \mathbf{R}$ и $W_{1}(x)$ - действительный и ограниченный дифференцируемая функция. Мы называем эти потенциалы $W$ - $d W$-потенииалами и подробно изучаем нелинейные режимы для NLS c W-dW-потенциалом. Мы показываем, что (i) эти режимы существуют только для малые амплитуды потенциала W-dW и являются только приближенными (псевдомоды); (ii) несмотря на этот факт, они устойчивы и демонстрируют динамику, которая описывается коллективным координатным подходом.

Ключевые слова: нелинейное уравнение Шрёдингера, нелинейные режимы, диссипация, четно-временная симметрия.

Recently Y. Kominis and coauthors [Chaos, Solitons and Fractals, 118, 222-233 (2019)] have suggested that Nonlinear Schrödinger Equation (NLS) with complex potential of the form $W(x)=W_{1}(x)+$ $i C W_{1, x}(x)$, supports the continuous families of localized nonlinear modes. Here $C \in \mathbf{R}$ and $W_{1}(x)$ is a real-valued and bounded periodic, quasiperiodic, etc.) differentiable function. We call these potentials $W-d W$ potentials and study in detail the nonlinear modes for NLS with W-dW potential. We show that (i) these modes exist only for small amplitudes of $\mathrm{W}-\mathrm{dW}$ potential and are approximative only (pseudo-modes); (ii) in spite of this fact, they are robust and exhibit the dynamics that is described by the collective coordinate approach. Keywords: nonlinear Schrödinger equation, nonlinear modes, dissipation, parity-time symmetry.

There are two cases when the nonlinear Schrödinger equation (NLSE) with an external complex potential $W(x)$,

$$
i \Phi_{t}=-\Phi_{x x}+W(x) \Phi \pm 2|\Phi|^{2} \Phi
$$

The work is supported by Russian Science Foundation (Grant No. 20-11-19995.)

Georgy Alfimov, PhD, MIET University (Moscow, Russia), professor;

Alexander Slobodyanyuk, MIET University (Moscow, Russia), master student;

Dmitry Zezyulin, PhD, ITMO University, (St-Petersburg, Russia), postdoc; 
supports continuous families of localized stationary modes of the form

$$
\begin{gathered}
\Phi(x, t)=\phi(x) e^{i \mu t}, \quad \mu \in \mathbf{R}, \\
\lim _{x \rightarrow \infty} \phi(x)=\lim _{x \rightarrow-\infty} \phi(x)=0 .
\end{gathered}
$$

They are (i) the $\mathcal{P} \mathcal{T}$-symmetric potentials and (ii) the Wadati potentials. Recently Y. Kominis and coauthors, [1], have suggested that the continuous families can be also found for complex potentials of the form

$$
W(x)=W_{1}(x)+i C W_{1, x}(x),
$$

where $C$ is an arbitrary real and $W_{1}(x)$ is a real-valued and bounded differentiable function.

For brevity, we call these potentials $W$ - $d W$ potentials. The results of our study for NLS with W-dW potentials are as follows.

(A) If $\mathrm{W}$-dW potential is small (of order $\varepsilon \ll 1$ ) our asymptotic procedure stops at the terms of the $\varepsilon^{2}$ order. So we conjecture that no continuous families of authentic nonlinear modes exist in this case, but pseudo-modes that satisfy the equation up to $\varepsilon^{2}$-error can indeed be found.

(B) For a W-dW potential well of finite depth we support the hypothesis of the point (A) with numerical arguments.

(C) The simulation of dynamics of the pseudo-modes show that if the amplitude of $\mathrm{W}-\mathrm{dW}$ potential is small, the pseudo-modes are robust and display persistent oscillations around the certain position predicted by the asymptotic expansion.

(D) We found the authentic stationary modes of (1) which do not form a continuous family, but exist as isolated points. Numerical simulations reveal dynamical instability of these stationary modes.

The details of our study can be found in [2].

\section{Литература}

1. Y. Kominis, J. Cuevas-Maraver, P. G. Kevrekidis et al, Continuous families of solitary waves in non-symmetric complex potentials: A Melnikov theory approach, Chaos, Solitons \& Fractals 118, 222, (2019).

2. D.A. Zezyulin, A.O. Slobodyanyuk, G.L. Alfimov, On existence of continuous families of stationary nonlinear modes for a class of complex potentials, arXiv:2104.11920. 